\title{
Reform der Primärversorgung in Österreich
}

\author{
Gibt es Potenzial im Bundesland Tirol?
}

\author{
Herbert Johann Bachler · Raphael Bertsch (D)
}

Eingegangen: 14. Juli 2017 / Angenommen: 16. November 2017 / Online publiziert: 11. Dezember 2017 (c) Der/die Autor(en) 2017. Dieser Artikel ist eine Open-Access-Publikation.

Zusammenfassung Die österreichische Bundes-Zielsteuerungskommission (BZK) beschloss im Jahr 2014 ein Konzept zur Reform des Primärversorgungssystems. Dadurch soll vor allem die Allgemeinmedizin gestärkt werden. Ziel der vorliegenden Studie war es, die bestehenden Formen der Zusammenarbeit in allgemeinmedizinischen Ordinationen Tirols zu ermitteln und einen Vergleich mit den Anforderungen an zukünftige Primärversorgungszentren zu erstellen. Dazu wurde mittels Online-Fragebogen eine Umfrage unter den niedergelassenen AllgemeinmedizinerInnen (AM) in Tirol durchgeführt. Diese Umfrage unter einem repräsentativen Kollektiv von 87 niedergelassenen AM ergab, dass nur ca. $20 \%$ der erhobenen Ordinationen das im Konzept beschriebene Kernteam bereits heute erfüllen. Unterschiede bei der Umsetzung von Anforderungsmerkmalen bestehen häufig in Abhängigkeit vom Stadt-Land-Verhältnis. Besonders unzufrieden sind die AM mit den Bereichen Administration, Finanzierung sowie Wertschätzung des Berufsbildes. Insgesamt besteht ein großer Wunsch nach Gruppenpraxen. Nur wenige AM gaben negative Erwartungshaltungen bezüglich Primärversorgungszentren an. Verbesserungswürdig erscheint die zeitliche Verfügbarkeit insbesondere am Tagesrand und in den Nachtstunden.

Schlüsselwörter Primärversorgung • Patientenzentrierung · Innovation · Primary Health Care · Interdisziplinarität

\footnotetext{
Dr. med. univ. H. J. Bachler

Tiroler Gesellschaft für Allgemeinmedizin (TGAM),

Medizinische Universität Innsbruck (MUI), Plattform

Allgemeinmedizin, Innsbruck, Österreich

R. Bertsch ( $\varangle)$

Medizinische Universität Innsbruck, Innsbruck, Österreich raphael.bertsch@student.i-med.ac.at
}

\section{Primary care reforms in Austria Is there potential in the region of Tyrol?}

Summary Within the Austrian health care reform, a new policy is generally proposed to strengthen primary care (PC). The aim of this study was to illustrate the status quo in the Tyrolean PC system and to provide the opportunity to examine the reform feasibility. Therefore, a prospective cross-sectional study was performed among licensed GPs in Tyrol, using an online questionnaire. The results of the survey among 87 GPs show that only $20 \%$ of Tyrolean PC settings already fulfill the coreteam availabilty described in the reformplans. However, there are differences between urban and rural areas. The highest dissatisfaction was detected in the fields of administration, financing and appreciation of PC. Primarily there is a request by the GPs for implementation of group practices. Only few concerns about the proposed PC centers were noticed. It seems necessary to improve availability, especially outside the core times and during the night.

Keywords Primary health care Patient-centred-care Innovation · Interdisciplinarity

\section{Einleitung}

In der Deklaration von Alma-Ata definiert sich der Begriff Primary Health Care (PHC) als erste Ebene, auf der die Bevölkerung mit dem Gesundheitssystem in Kontakt tritt [1]. Im Mittelpunkt steht eine kontinuierliche Betreuung und individuelle Anpassung von Therapieempfehlungen. Dadurch soll ein gesundheitsfördernder Effekt erzielt werden [2-4]. Ein besonderes Augenmerk liegt auf der Krankheitsprävention und der Behandlung von chronischen Erkrankungen [4]. Internationale Studien legen eine positive Korrelation zwischen gut ausgebildeten All- 


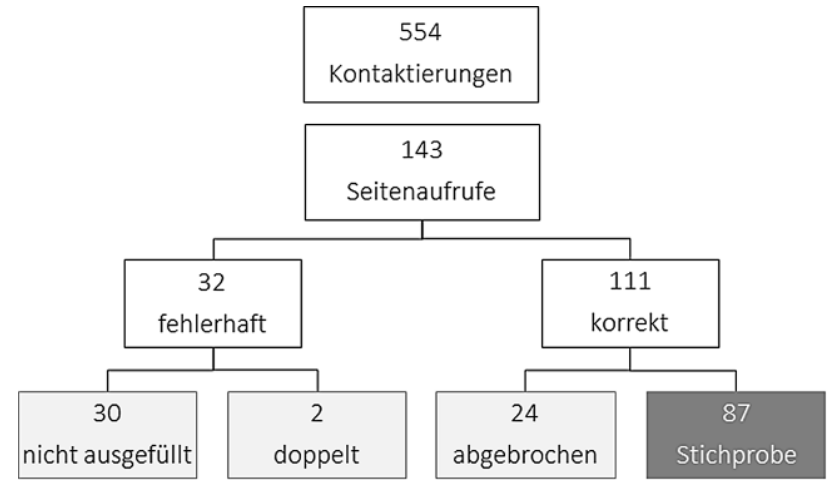

Abb. 1 Prozessablauf zur Auswertung

gemeinmedizinerInnen (AM) mit Schwerpunkt im Bereich Familienmedizin und einer höheren klinischen Effizienz in Ländern mit ausgeprägten Primärversorgungssystemen nahe $[5,6]$.

In Zusammenschau der Vorteile für die Bevölkerung wurde auch in Österreich im August 2017 ein Reformgesetz der Primärversorgung auf Basis des Konzeptes der Bundes-Zielsteuerungskommission (BZK) aus dem Jahr 2014 beschlossen [7-9]. Insgesamt gilt die Ausprägung von PHC hierzulande als schwach und der Stellenwert von AM als erste Kontaktinstanz als relativ patientenabhängig $[4,6,10,11]$. Ein fundamentaler Reforminhalt ist die multiprofessionelle Zusammenarbeit mit anderen Gesundheits- und Sozialberufen sowie die interdisziplinäre Zusammenarbeit von AM mit ÄrztInnen anderer Fachdisziplinen, mit dem Ziel eine ganzheitliche und kontinuierliche Patientenbetreuung zu erreichen. Teamzusammenarbeit im engeren Sinne sollte sowohl ortsübergreifend, als auch an einem gemeinsamen Standort möglich sein. Die Bildung dieser Teams soll in der Folge auch beteiligte Gesundheitsberufe entlasten und deren Work-LifeBalance verbessern [12].

Um die Reforminhalte in der Praxis zur Umsetzung bringen zu können, erscheint zunächst die Kenntnis regional vorherrschender Strukturen essenziell. Das Ziel dieser Studie war es daher, durch die Erhebung vorhandener Organisationsstrukturen und Arbeitsweisen allgemeinmedizinischer Ordinationen in Tirol eine Forschungslücke zu füllen. Die Orientierung der Umfrage am Konzept der BZK sollte einen Vergleich der zukünftigen Anforderungen mit dem Status quo ermöglichen. Ein Überblick über benötigte Unterstützungsmöglichkeiten wurde durch die Erhebung von Zufriedenheit und Zukunftswünschen der AM aufgezeigt. Letztlich erfolgte die Prüfung der Korrelation zwischen einem speziell erstellten Anforderungsscore, welcher die im Reformkonzept enthaltenen Themen umfasste, und dem ebenfalls speziell errechneten Zufriedenheitscore.

\section{Methoden \\ Studiendesign und Rekrutierung}

Die Studie wurde als prospektive Querschnittsstudie mittels Online-Umfrage unter den niedergelassenen AM in Tirol in der Zeit von Juni bis Oktober 2016 durchgeführt. Nach der ersten Kontaktaufnahme via E-Mail erfolgten im Verlauf auf gleichem Wege zwei Erinnerungen zur Teilnahme. Insgesamt waren zum Zeitpunkt der Studie 572 AM ( $w=174 ; \mathrm{m}=398)$ in der Liste der Ärztekammer für Tirol (ÄKT) verzeichnet. Nur 553 Personen waren tatsächlich via E-Mail erreichbar. Es wurden 143 Seitenaufrufe verzeichnet. Wie in Abb. 1 dargestellt, erfolgte anschließend eine Prüfung dieser Aufrufe, sodass schlussendlich 87 vollständig ausgefüllte Fragebögen als Stichprobe herangezogen wurden. Die Rücklaufquote betrug 15,7\%. Verglichen mit dem Kollektiv zeigt die Stichprobe eine ähnliche Verteilung von Geschlecht, Altersgruppe und Praxisform, wenngleich geringfügig mehr männliche und tendenziell eher jüngere $\mathrm{AM}$ an der Umfrage teilnahmen.

\section{Fragebogen}

Im Vorfeld der Fragebogengestaltung wurde eine selektive Literaturrecherche durchgeführt und die erhobenen Informationen in den Prozess der Fragebogengestaltung integriert [10, 13-16]. Neben Fragen zu soziodemografischen Merkmalen umfasste die Erhebung insgesamt 15 Fragegruppen zu den Aspekten Erreichbarkeit, Verfügbarkeit, Interdisziplinarität, Arbeitsweise und Zufriedenheit bzw. Zukunftswünsche. Nach einem Pre-Test $(n=17)$ wurden keine weiteren Änderungen am Fragebogen vorgenommen. Der Leitfaden zur Fragebogengestaltung ist in Abb. 2 dargestellt.

\section{Erreichbarkeit}

Ein niederschwelliger Zugang für alle Bevölkerungsgruppen ist ein zentraler Punkt im Konzept der BZK. Im Rahmen der Umfrage wurde daher einerseits die Erreichbarkeit mittels öffentlicher Verkehrsmittel und andererseits die Barrierefreiheit erhoben.

\section{Verfügbarkeit}

Im Konzept der BZK werden die Öffnungszeiten von Montag bis Freitag jeweils zwischen 7.00 Uhr und 19.00 Uhr angegeben. Zusätzlich sollen für Notfälle auch außerhalb dieser Zeiten Bereitschaftsdienste verfügbar sein. Für PatientInnen mit eingeschränkter Mobilität, ist die Möglichkeit eines Hausbesuches vorgesehen [12]. Die AM wurden im Rahmen der Umfrage zu regulären Öffnungszeiten sowie zur Anzahl von Hausbesuchsstunden pro Woche befragt. 
Abb. 2 Leitfaden zur Erstellung des Fragebogens
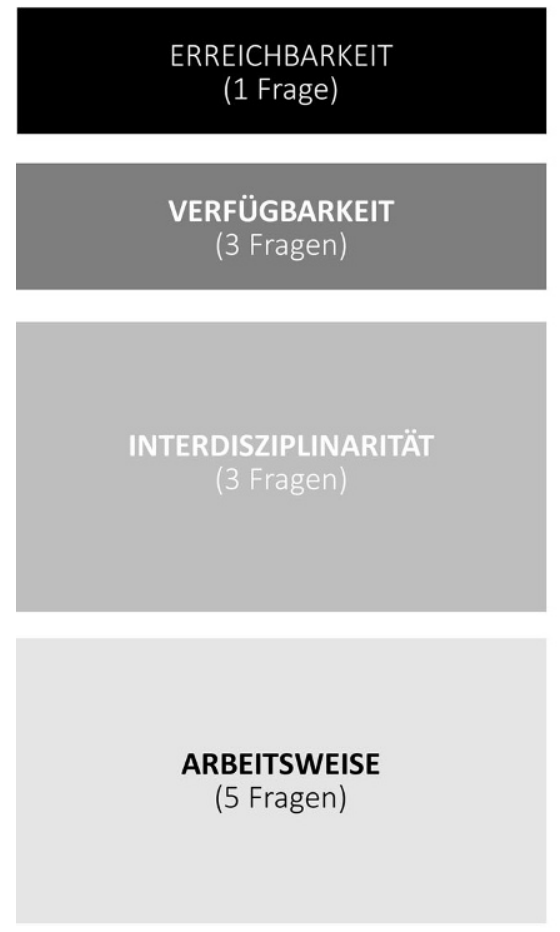

MEINUNG

(2 Fragen)

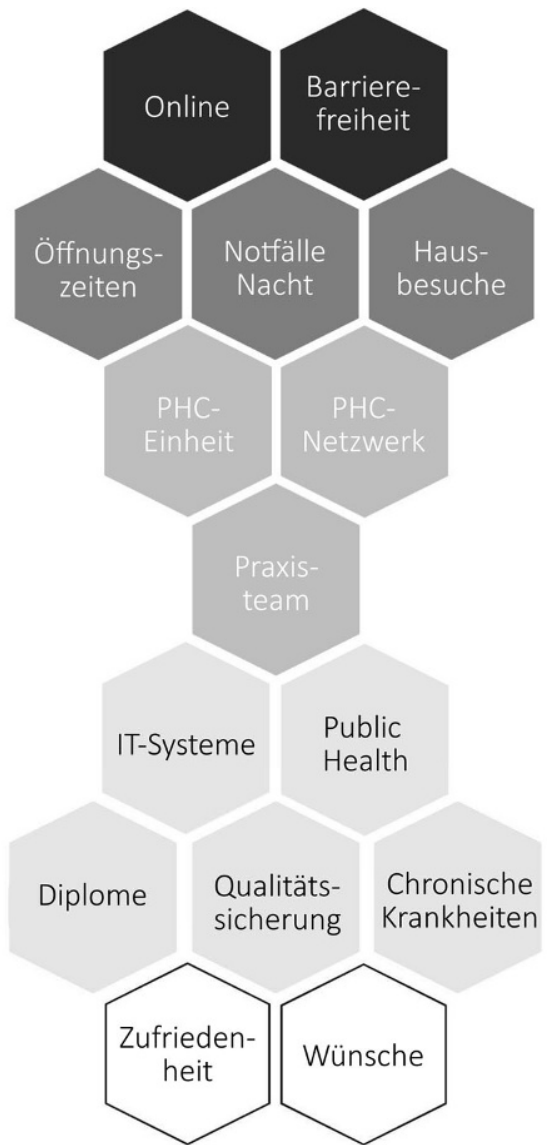

\section{Interdisziplinarität und Multiprofessionalität}

Ein wesentliches Merkmal des Reformkonzeptes ist die Zusammenarbeit von Gesundheitsberufen und ÄrztInnen in der Primärversorgung. Beschrieben werden zentrumsähnliche „PHC-Einheiten“ und ortsübergreifende „PHC-Netzwerke“. In Anlehnung an diese Definitionen wurden auch in der Umfrage die Zusammensetzung von Ordinationsteams und die Häufigkeit der Zusammenarbeit mit externen Gesundheits- oder Sozialberufen erhoben.

Anhand der angegebenen Berufsgruppen erfolgte, ähnlich wie bei Hoffmann et al. [10], folgende Modelleinteilung:

- Modell 1:AM (+ OrdinationsassistentIn)

- Modell 2: AM (+ OrdinationsassistentIn) + mind. 1 weitere Berufsgruppe

- Modell 3: AM (+ OrdinationsassistentIn) + mind. 2 weitere Berufsgruppen

Das Ausmaß der aktuell bestehenden Zusammenarbeit zwischen AM und anderen ÄrztInnen bzw. Angehörigen anderer Gesundheitsberufe wurde mittels 5-Punkt-Likert-Skala (nie, sehr selten/jährlich, selten/halbjährlich, gelegentlich/monatlich, häufig/ wöchentlich, sehr häufig/täglich) erfasst. Zudem wurde auch die räumliche Nähe anderer Gesundheitsund Sozialberufe zur Ordination der AM mittels Frei- text erfragt, welche im Folgenden als „co-location“ [17] bezeichnet wird.

\section{Arbeitsweise}

Der Austausch von Daten unter den GesundheitspartnerInnen wird als essenziell für ein funktionierendes Netzwerk angesehen [18]. Dazu wurde erhoben, ob ITInfrastruktur für Dokumentation, Informationsweitergabe, Erinnerungssysteme (z. B. für chronisch Kranke bzw. Impferinnerung) oder telemedizinische Dienste verwendet wird. Außerdem wurden die AM nach der Beteiligung an öffentlichen Gesundheitsaufgaben sowie der Qualitätssicherung, etwa durch Evaluierung mittels Feedbackerhebungen, befragt.

\section{Zufriedenheit/Zukunftswünsche}

Zur Erhebung der Zufriedenheit mit dem aktuellen System wurde die Meinung der AM zu den Punkten Attraktivität der Tätigkeitsfelder, Arbeits- und Rahmenbedingungen (Work-Life-Balance), Finanzielle Entlohnung und Honorierungsmodell, Gesellschaftlicher Status als AM und Ausmaß administrativer Tätigkeiten ermittelt. Die Auswahl dieser Kriterien erfolgte in Anlehnung an eine österreichweite Erhebung von Hoffmann et al. [19]. Ergänzt wurden diese Kriterien durch die Erhebung der Zufriedenheit mit dem Ausmaß der Zusammenarbeit mit anderen Gesundheitsberufen und der Erreichbarkeit der Allgemein- 
medizin für die Bevölkerung. Dies sind grundlegende Punkte im Konzept der BZK [9]. Als Skalierung wurde das österreichische Schulnotensystem mit fünf Stufen von sehr gut bis nicht genügend verwendet. Durch Freitextfelder wurden Verbesserungsvorschläge und Wünsche der AM für die Zukunft erhoben.

\section{Scoreentwicklung}

Im Rahmen der Studie wurden zwei Scores entwickelt, welche letztlich miteinander auf Korrelationen geprüft wurden. Zur Messung der Ausprägung der im Konzept der BZK geforderten Strukturmerkmale wurde der Anforderungsscore erstellt. Die Punkteverteilung wurde dabei so gewählt, dass auf die vier Indikatoren Erreichbarkeit, Verfügbarkeit, Interdisziplinarität und Arbeitsweise jeweils die gleiche Punktemenge entfiel. Zur Vergleichbarkeit der Werte innerhalb des Scores wurde eine Transformation der einzelnen Indikatoren auf den Zahlenbereich 0 bis 1 durchgeführt.

Zur Darstellung der Zufriedenheit von AM in Tirol wurde der Zufriedenheitsscore gewählt. Dieser Score

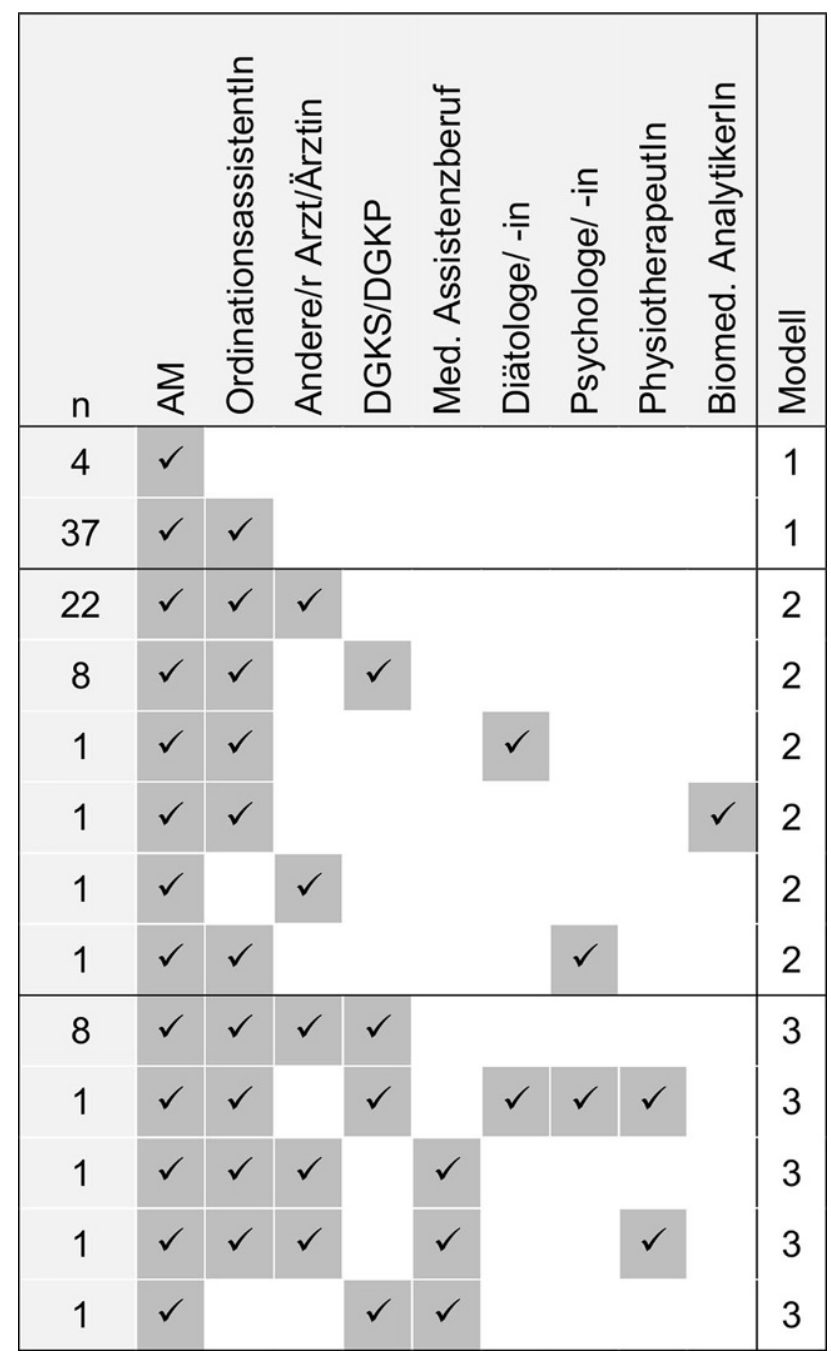

Abb. 3 Kombinationen von Berufsgruppen in Ordinationsteams setzt sich aus dem Durchschnitt der Punktesumme der vergebenen Schulnoten für alle erhobenen Bereiche der Zufriedenheit zusammen. Bei sieben erhobenen Zufriedenheitskategorien konnte somit eine maximale Notensumme von 35 erreicht werden. Die Bewertungsgrade wurden invertiert. Das bedeutet, dass beispielsweise die Angabe „Sehr gut“ mit 5 Punkten und die Angabe „Nicht genügend“ mit 1 Punkt im Score bewertet wurde.

\section{Datenanalyse}

Die Auswertung und statistische Analyse der Ergebnisse erfolgte nach deskriptiven Gesichtspunkten mittels SPSS Statistics 24. Insgesamt hatte die Auswertung der Daten einen explorativen Charakter. Fallweise wurden auffällige Daten zur Bestätigung zusätzlich mittels statistischer Tests wie dem Chi2-Test, Kruskal-WallisTest, Mann-Whitney-U-Test oder Korrelationsanalysen überprüft. Dabei wurde ein Signifikanzniveau von fünf Prozent gewählt. Bei Verwendung von LikertSkalen wurden die jeweiligen Antworten in Zahlen umgerechnet. Zusätzlich zum Chi2-Test wurden ggf. die Verteilungen mittels $z$-Test verglichen und mit der Bonferroni-Methode für Mehrfachvergleiche korrigiert. Anhand der angegebenen Einwohnerzahl der Standortgemeinde wurde eine Dichotomisierung (bis 8000 EinwohnerInnen = ländlich; ab 8001 EinwohnerInnen = städtisch/urban) vorgenommen.

\section{Ethikkomission}

Es wurde kein Votum der Ethikkommission benötigt, da es sich bei der Erhebung um keine patientenbezogenen Daten handelte.

\section{Ergebnisse}

\section{Erreichbarkeit}

Hinsichtlich der Erreichbarkeit zeigen sich zum Teil deutliche Unterschiede zwischen urbanen und ländlichen Regionen. Auffällig erscheint der Stadt-LandVergleich etwa bei der Erreichbarkeit mittels öffentlicher Verkehrsmittel. Hierbei ist der urbane Bereich ( $100 \% ; n=32$ ) dem ländlichen (84\%; $n=43$ ) überlegen. Gegenteilig ist diese Diskrepanz im barrierefreien Zugang. Während ein solcher am Land bei über $90 \%$ $(n=46)$ der Ordinationen besteht, ist es in den Städten nur bei knapp $44 \%(n=14)$ der Fall.

\section{Interdisziplinarität}

Für die Zusammensetzung des Ordinationsteams konnten die in Abb. 3 dargestellten Varianten erhoben werden. Die häufigste Kombination stellen AM mit OrdinationsassistentInnen dar $(n=37)$. Es zeigt sich, dass die Modelle $2(n=34)$ und $3(n=11)$ zusammen häufiger vorkommen als die Einzelpraxis, also 


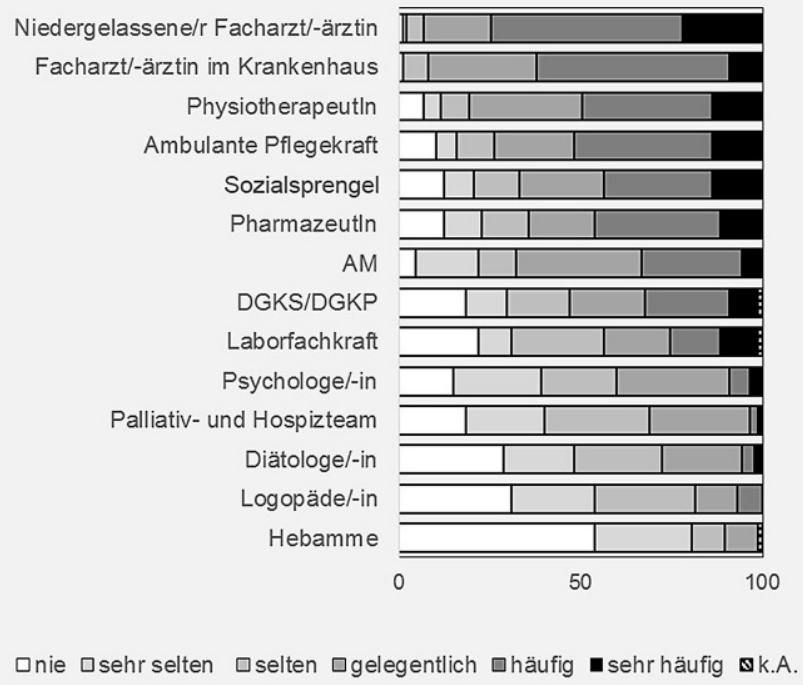

Abb. 4 Häufigkeit der Zusammenarbeit von AM mit anderen Gesundheitsberufen

Modell $1(n=41)$. Das Kernteam aus dem Konzept der BZK wurde nur in ca. $20 \%(n=17)$ der Ordinationen gefunden.

Zusammenarbeit mit Gesundheits- und Sozialberufen, die nicht Teil des eigentlichen Ordinationsteams sind, findet am häufigsten zwischen AM und niedergelassenen FachärztInnen (FÄ) oder FÄ in Krankenanstalten statt. Bei der Kooperation mit nichtärztlichen Gesundheitsberufen erreichten PhysiotherapeutInnen die höchsten Nennungen, gefolgt von ambulanten Pflegekräften. Am seltensten gaben die befragten AM Zusammenarbeit mit LogopädInnen bzw. Hebammen an. Grafisch sind die Zusammenarbeitshäufigkeiten in Abb. 4 dargestellt.

Die Auswertung der sogenannten „co-location“ [17] zeigt, dass etwa $60 \%(n=51)$ der Befragten keine der in Abb. 5 dargestellten Varianten von räumlicher Nähe mit anderen Berufen besitzen. Die meisten räumlichen Nahbeziehungen bestehen zu niedergelassene FÄ sowie PhysiotherapeutInnen (jeweils $n=22$ ).

\section{Verfügbarkeit}

Im Durchschnitt haben die Ordinationen pro Woche ca. 23,5 h geöffnet, wobei sich ein deutlicher Fokus auf den Vormittagen zeigt. Die im Konzept der BZK [12] definierten Kernzeiten werden nur selten erfüllt. In Abb. 6 sind die Öffnungszeiten farblich dargestellt, wobei optisch die Tagesrandzeiten sowie die Mittagszeit als relativ schwach ausgeprägt imponieren. Im Wochenverlauf zeigen sich geringe Öffnungszeiten am Freitagnachmittag. An Samstagen gaben nur drei der befragten $\mathrm{AM}$ an, regulär geöffnet zu haben. Im Stadt-Land-Vergleich wurden in urbanen Regionen ( $M=23,6)$ minimal höhere Stundenzahlen pro Woche angegeben als in ländlichen $(M=23,3)$. Demgegenüber wurden auf dem Land $(M=5,2)$ durchschnittlich

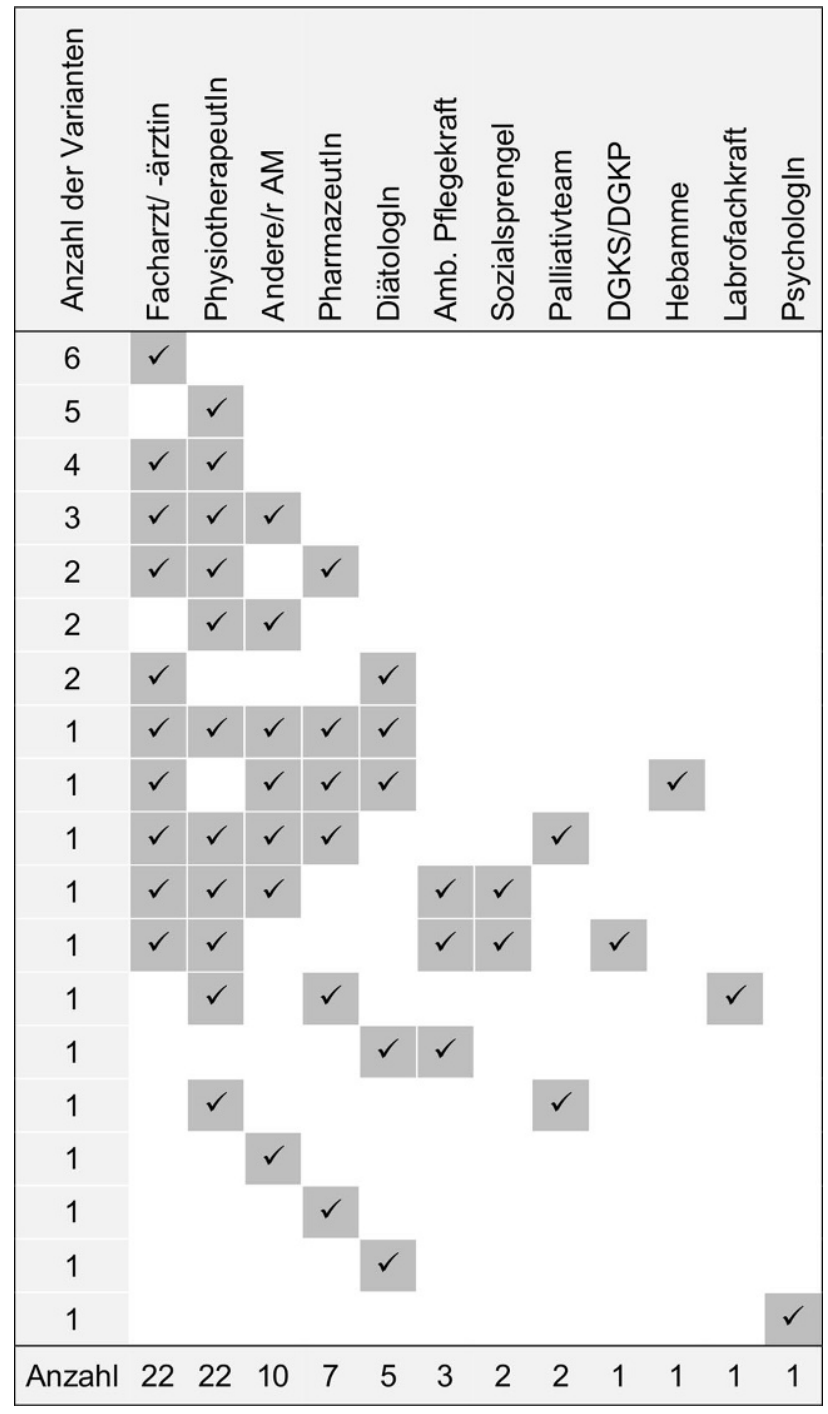

Abb. 5 Erhobene Berufsgruppen mit räumlicher Nähe zu allgemeinmedizinschen Ordinationen

mehr Hausbesuchsstunden pro Woche genannt als in der Stadt $(M=4,0)$. Verfügbarkeit via Mobiltelefon bei Notfällen und in der Nacht bestand bei $52 \%(n=45)$. Ein hausärztlicher Nachtdienst im Sprengel wurde von $48 \%(n=42)$ der AM genannt, wobei dies signifikant häufiger bei ländlichen Ordinationen $(71 \%, n=$ 36) zutraf als bei städtischen $(9 \%, n=3)$.

\section{Arbeitsweise}

Elektronische Dokumentation findet derzeit in ca. $80 \%$ ( $n=70)$ der befragten Ordinationen statt. Teilweise etabliert sind darüber hinaus Möglichkeiten zur elektronischen Weitergabe von Informationen an andere ÄrztInnen ( $49 \%$; $n=43$ ), computerbasierte Kommunikation innerhalb der Praxis $(46 \%, n=40)$ sowie Erinnerungssysteme für PatientInnen (27\%; $n=24)$. Eher selten kommt demgegenüber die Nutzung von Telemedizin vor $(n=10)$. Keine der befragten Ordina- 


\begin{tabular}{|c|c|c|c|c|c|c|c|c|c|c|c|c|c|c|c|c|c|c|c|c|c|c|c|c|c|c|}
\hline Uhrzeit & 6.00 & & 7.00 & & 8.00 & & 9.00 & & 10.00 & & 11.00 & & 12.00 & & 13.00 & & 14.00 & & 15.00 & & 16.00 & 17.00 & 18.00 & 19.00 & & 20.00 \\
\hline Montag & 0 & 0 & 3 & 12 & 61 & 70 & 73 & 74 & 74 & 73 & 67 & 56 & 13 & 8 & 5 & 2 & 6 & 7 & 16 & 19 & 41 & 48 & 24 & 0 & 0 & 0 \\
\hline
\end{tabular}

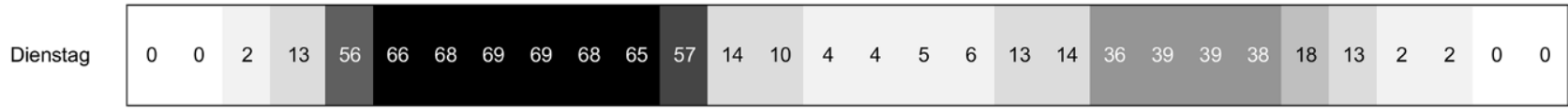

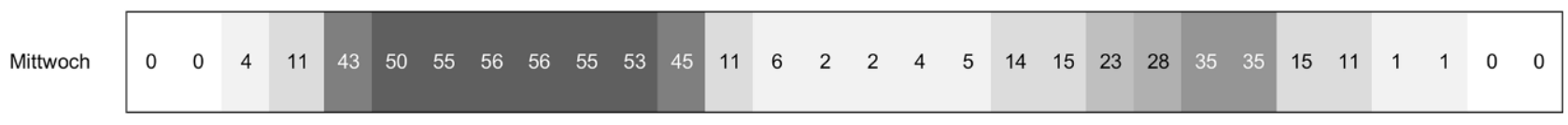

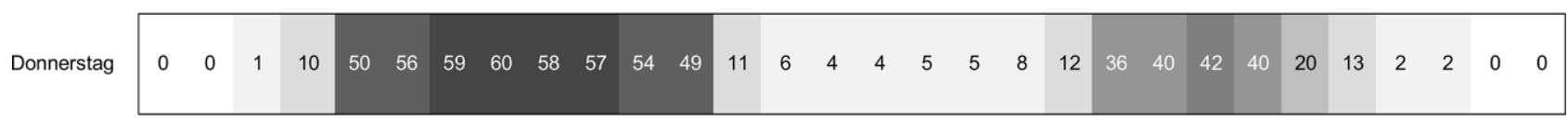

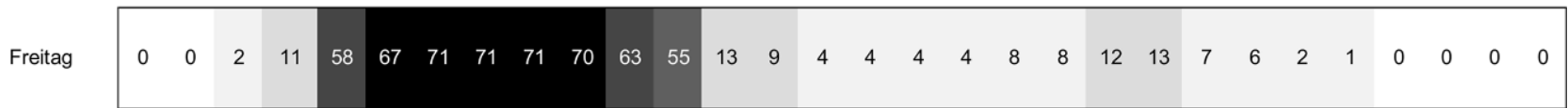

\begin{tabular}{l|lllllllllllllllllllllllllllllllllllll} 
Samstag & 0 & 0 & 0 & 0 & 0 & 0 & 2 & 3 & 3 & 3 & 1 & 0 & 0 & 0 & 0 & 0 & 0 & 0 & 0 & 0 & 0 & 0 & 0 & 0 & 0 & 0 & 0 & 0 & 0 & 0
\end{tabular}

Abb. 6 Erhobene Öffnungszeiten allgemeinmedizinischer Ordinationen

tionen stimmt ihr IT-System zur Kompatibilität mit anderen Ordinationen ab.

Etwa $59 \%(n=51)$ der befragten AM gaben an, sich an öffentlichen Gesundheitsaufgaben zu beteiligen. Davon nehmen $45 \%(n=39)$ an Vorsorge- und Screeningprogrammen teil. Gruppenprojekte, wie etwa Herzsport- oder Selbsthilfegruppen, kamen nur bei $6 \%(n=5)$ der Befragten vor. Im Stadt-Land-Vergleich fällt auf, dass die Beteiligung an öffentlichen Gesundheitsaufgaben insgesamt am Land deutlich häufiger (73\%; $n=37$ ) angegeben wurde als in der Stadt (12\%; $n=12$ ). Ein Signifikanztest bestätigte diesen Unterschied (Chi2 = 9,988, $p=0,002, \mathrm{df}=1$ ).

In Bezug auf die Qualitätssicherung kann festgestellt werden, dass interne Fallbesprechungen ( $n=$ 64), Fortbildungen ( $n=59$ ), Kompetenzverteilung im Praxisteam ( $n=65$ ) und das interne Qualitäts- und Fehlermanagement ( $n=53$ ) am häufigsten bestanden. In etwa der Hälfte der Fälle wurde die Verwendung von evidenzbasierten Leitlinien $(n=48)$ genannt sowie von ca. einem Drittel die Teilnahme an Qualitätszirkeln $(n=48)$. Selten kamen Feedbackerhebungen $(n=2)$ und Standardisierung beim Behandlungs- oder Krankenhausentlassungs-Management $(n=19)$ vor.

\section{Zufriedenheit/Zukunftswünsche}

Als Schwerpunkt der Studie kann die Erhebung der Zufriedenheit und der Wünsche niedergelassener AM in Tirol bezeichnet werden. In Abb. 7 ist die Verteilung der Zufriedenheitsnoten für die einzelnen Bereiche dargestellt. Während die Bereiche Verfügbarkeit der Allgemeinmedizin für die Bevölkerung, Attraktivität der Tätigkeitsfelder und gesellschaftlicher Status als AM relativ gut bewertet wurden, zeigen die AM mit der finanziellen Entlohnung und dem Honorierungsmodell sowie dem Administrationsaufwand geringe Zufriedenheit.

Die Erhebung der individuellen Wünsche, die von insgesamt 38 befragten AM angegeben wurden, ergab, dass besonders die Erleichterung von Gemeinschaftspraxen und Vertretungsregelungen sowie die Steigerung des Vertrauens durch die Stakeholder (je $n=14$ ) von Bedeutung sind. Als ähnlich wichtig wurden der Abbau von Bürokratie sowie eine bessere Entlohnung ohne Honorardeckelung (je $n=12$ ) genannt. Weitere Wünsche bezogen sich auf die Verbesserung des Leistungskataloges von Krankenkassen und der Ausbildung von angehenden AM mit mehr Praxisbezug sowie eine familienfreundlichere Strukturierung der Allgemeinmedizin (je $n=5$ ). Der Wunsch nach der Erleichterung von Hausapotheken wurde in vier Fällen genannt. Jeweils zweimal wurden mehr Hilfestellung durch die Ärztekammer und mehr Kassenstellen

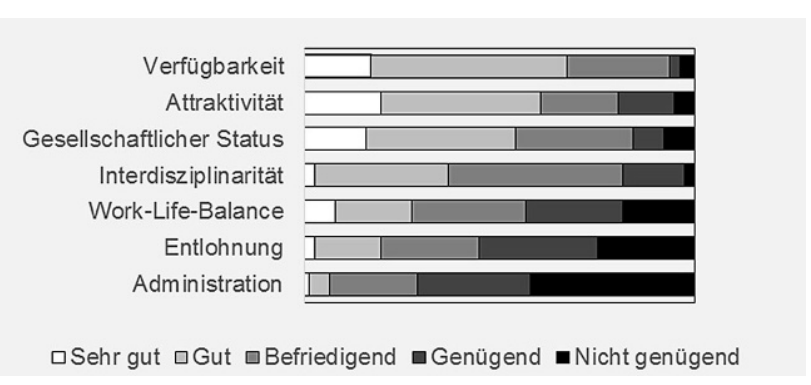

Abb. 7 Zufriedenheit der AM mit dem bestehenden Primärversorgungssystem 
Tab. 1 Errechnete Mittelwerte (M) für die Indikatoren des Anforderungsscores

\begin{tabular}{|l|l|l|l|l|} 
& Mittelwert (M) & Standardabweichung (SD) & Minimum & Maximum \\
\hline Erreichbarkeit & 0,745 & 0,171 & 0,20 & 1,00 \\
\hline Interdisziplinarität & 0,550 & 0,244 & 0 & 0,99 \\
\hline Verfügbarkeit & 0,325 & 0,019 & 0 & 0,80 \\
\hline Arbeitsweise & 0,405 & 0,022 & 0 & 0,75 \\
\hline
\end{tabular}

Abb. 8 Korrelationsanalyse zwischen Anforderungsscore und Zufriedenheitsscore

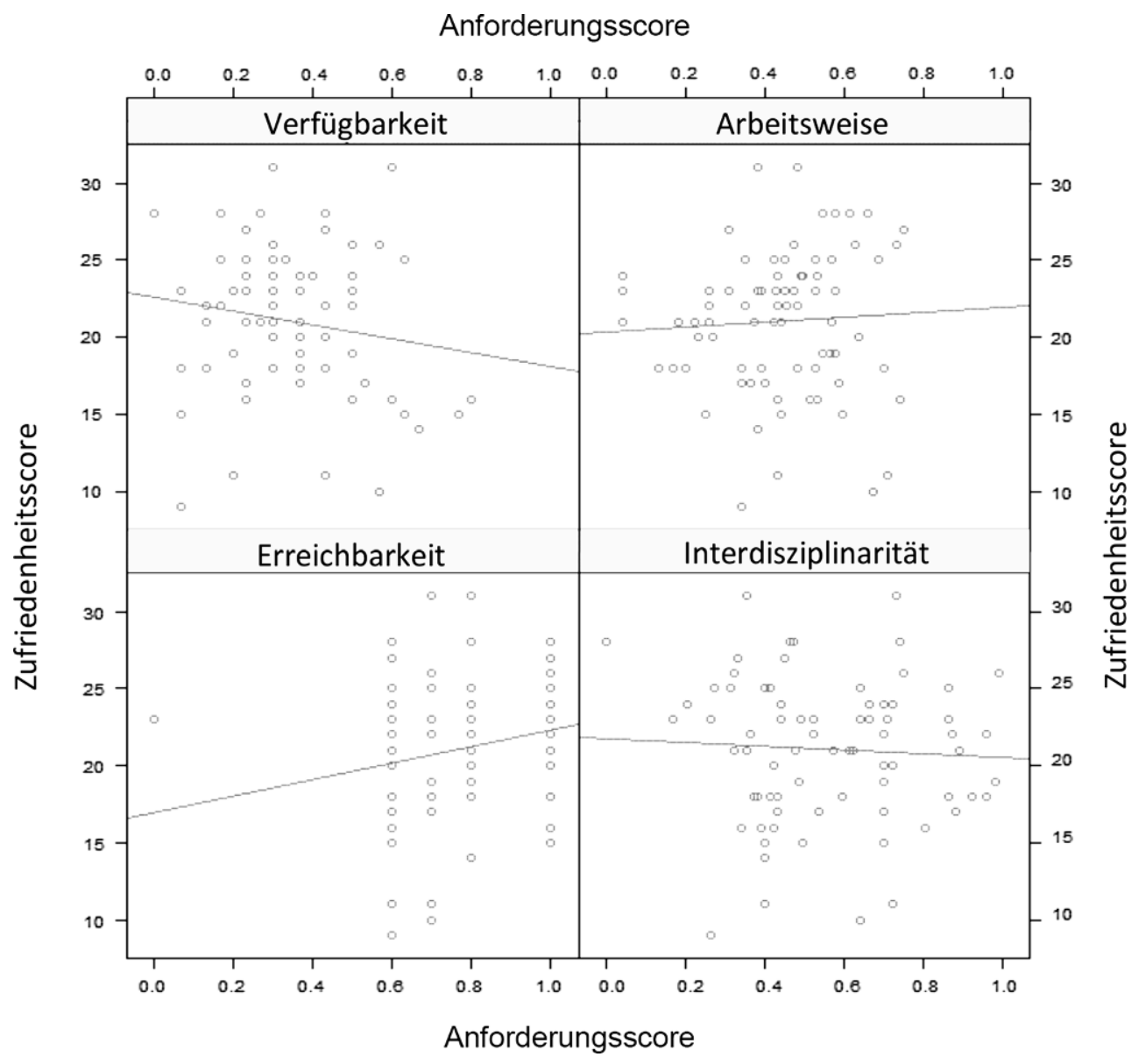

für FachärztInnen erwähnt. Lediglich in zwei Fällen äußerten die AM Bedenken gegenüber PHC-Zentren.

\section{Anforderungsscore}

Für die Implementierung des Anforderungsscores wurden 83 der 87 Befragten eingeschlossen. Der maximal erreichbare Wert wurde lediglich für den Indikator Erreichbarkeit erzielt. Aus Tab. 1 kann abgelesen werden, dass für diese Variable auch der Mittelwert am höchsten ausgefallen ist $(\mathrm{M}=0,745)$. Den zweithöchsten Mittelwert $(M=0,550)$ konnten die AM im Bereich Interdisziplinarität erreichen. Geringere Werte wurden in den Bereichen Verfügbarkeit $(\mathrm{M}=$ $0,405)$ und Arbeitsweise $(M=0,325)$ verzeichnet. Zur Überprüfung eines möglichen Zusammenhanges zwischen Zufriedenheitsscore und Anforderungsscore wurde eine Korrelationsanalyse durchgeführt. Abb. 8 zeigt die grafische Darstellung der Ergebnisse. Die statistische Auswertung ergibt keine signifikanten Ergebnisse.

\section{Diskussion/Schlussfolgerungen}

Die durchgeführte Studie stellt die erste Datenerhebung dieser Art in Tirol dar. Der Umfang der Erhebung kann aufgrund des großen Anteils am Gesamtkollektiv durchaus als Stärke dieser Studie angesehen werden. Darüber hinaus orientierte sich die Erstellung des Fragebogens weitestgehend am Konzept der BZK, was einen Vergleich der Ist-Situation mit den Reforminhalten möglich machen soll. Dennoch sei erwähnt, dass es sich lediglich um eine Stichprobe handelt und tendenziell eher männliche bzw. jüngere AM in dieser vertreten waren. Anhand des Ergebnisses allgemein gültige Aussagen $\mathrm{zu}$ treffen, ist demnach eine Limitation dieser Studie, wenngleich sie durchaus einen 
Einblick in vorhandene Primärversorgungsstrukturen in Tirol liefern kann.

Es lässt sich feststellen, dass seitens der AM großteils der Wunsch besteht, Gemeinschafts- und Gruppenpraxen zu implementieren. In diesem Bereich entsprechen die Wünsche der AM auch weitgehend den Reforminhalten. Die aktuell vorherrschende Situation zeigt jedoch, dass das im Konzept der BZK beschriebene Kernteam derzeit bei weniger als $20 \%$ der allgemeinmedizinischen Ordinationen vorhanden ist.

Während die ordinationsübergreifende Zusammenarbeit mit FÄ, PhysiotherapeutInnen, ambulanten Pflegekräften, Sozialsprengel, PharmazeutInnen, anderen AM und Laborkräften bereits relativ häufig stattfindet, ist vor allem die Kooperation mit Hebammen, LogopädInnnen, DiätologInnen, Palliativ- und Hospizteams sowie PsychologInnen unterrepräsentiert. Ein möglicher Grund dafür könnte eine geringe Relevanz der Zusammenarbeit im Alltag sein. Die Frage, ob zukünftig der Bedarf für multiprofessionelle Teams und Netzwerke mit den derzeit selten vertretenen Berufsgruppen bestehen könnte, wäre ein mögliches weiterführendes Forschungsfeld.

Verglichen mit den Anforderungen im Konzept der BZK unterscheiden sich speziell in den Tagesrandzeiten die aktuellen Öffnungszeiten der erhobenen Ordinationen relativ stark. Nur wenige Ordinationen sind bereits ab $7.00 \mathrm{Uhr}$ bzw. bis 19.00 geöffnet und speziell in den Mittagsstunden kann die Verfügbarkeit durchaus als gering bezeichnet werden. Zur Umsetzung der Anforderungen im Konzept wäre vermutlich eine bessere Abstimmung der Öffnungszeiten zwischen den Ordinationen sinnvoll. Somit könnten Lücken, wie sie derzeit bestehen, gefüllt und die Verfügbarkeit der AM für die Bevölkerung verbessert werden.

In der Korrelationsanalyse zeigen sich bei höheren Werten des Indikators Verfügbarkeit tendenziell eher niedrigere Werte des Zufriedenheitsscores. Dies könnte möglicherweise mit einer höheren Zahl an Arbeitsstunden zusammenhängen, welche folglich die geringere Zufriedenheit erklärt. Anders ist die Tendenz beim Indikator Erreichbarkeit. Jene AM, die hohe Werte für Barrierefreiheit bzw. Erreichbarkeit mittels öffentlicher Verkehrsmittel oder via E-Mail erreichten, hatten tendenziell auch höhere Zufriedenheitsscores. Die Korrelationen für die Indikatoren Arbeitsweise und Interdisziplinarität mit dem Zufriedenheitssocre waren äußerst gering, weshalb eine weitere Diskussion wenig sinnvoll erscheint.

Zusammenfassend lässt sich feststellen, dass seitens der befragten AM durchaus der Wunsch nach Gemeinschaftspraxen besteht, die Ausprägung dieser, zumindest in Bezug auf das Kernteam aus dem Konzept der BZK, derzeit jedoch noch gering ist. Die größte Unzufriedenheit unter den AM besteht bezüglich des Administrationsaufwands sowie der Finanzierung und des zugrundeliegenden Honorierungsmodelles. Diese Punkte haben, aus Sicht der befragten AM, gemeinsam mit einer Steigerung des Vertrauens und der Wertschätzung sowie der Schaffung von besseren Vertretungsregelung oberste Priorität zur Umsetzung.

Funding Open access funding provided by University of Innsbruck and Medical University of Innsbruck.

Interessenkonflikt H.J. Bachler und R. Bertsch geben an, dass kein Interessenkonflikt besteht.

Open Access Dieser Artikel wird unter der Creative Commons Namensnennung 4.0 International Lizenz (http:// creativecommons.org/licenses/by/4.0/deed.de) veröffentlicht, welche die Nutzung, Vervielfältigung, Bearbeitung, Verbreitung und Wiedergabe in jeglichem Medium und Format erlaubt, sofern Sie den/die ursprünglichen Autor(en) und die Quelle ordnungsgemäß nennen, einen Link zur Creative Commons Lizenz beifügen und angeben, ob Änderungen vorgenommen wurden.

\section{Literatur}

1. World Health Organization. Declaration of Alma-Ata. AlmaAta. 1978. http://www.euro.who.int/_data/assets/pdf_ file/0009/113877/E93944.pdf?ua=1. Zugegriffen: 1. Nov. 2017.

2. Shi L. The impact of primary care: a focused review. Scientifica (Cairo). 2012; 2012. https://doi.org/10.6064/2012/ 432892.

3. Van Loenen T, Faber MJ, Westert GP, Van den Berg MJ. The impact of primary care organization on avoidable hospital admissions for diabetes in 23 countries. Scand JPrim Health Care. 2016;34(1):5-12. https://doi.org/10.3109/02813432. 2015.1132883.

4. WorldHealth Organization. Buildingprimarycareinachanging Europe. 2015. http://www.euro.who.int/_data/ assets/pdf_file/0018/271170/BuildingPrimaryCareChang ingEurope.pdf.Zugegriffen: 4. Mai 2017.

5. WONCA. The European definition of General Practice/ Family Medicine. 2011. http://www.woncaeurope.org/ sites/default/files/documents/Definition 3rd ed 2011 with revised wonca tree.pdf. Zugegriffen:4. Mai 2017.

6. Stigler FL, Starfield B, Sprenger M, Salzer HJ, Campbell SM. Assessing primary care in Austria: room for improvement. Fam Pract. 2013;30(2):185-9. https://doi.org/10. 1093/fampra/cms067.

7. Hofmarcher MM. The Austrian health reform 2013 is promising but requires continuous political ambition. Health Policy.2014;118(1):8-13.https://doi.org/10.1016/j.healthpol. 2014.09.001.

8. Hofmarcher MM, Rack HM. Health care systems in transition:Austria 2006. Health Syst Transit. 2006;8(3):1-247.

9. Gesundheitsreformumsetzungsgesetz. In: BGBl. I Nr. 131/ 2017. 2017. http://www.ris.bka.gv.at/Dokumente/Bgbl Auth/BGBLA_2017_I_131/BGBLA_2017_I_131.pdf. Zugegriffen: 1. Nov. 2017.

10. Hoffmann K, George A, DornerTE, SüßK, SchäferWL, Maier M.Primaryhealthcareteams putto thetestacross-sectional study from Austria within the QUALICOPC project. BMC Fam Pract. 2015;16:168. https://doi.org/10.1186/s12875015-0384-9.

11. Huter S. Die Rolle der AllgemeinmedizinerInnen als erste Anlaufstelle im österreichischen Gesundheitssystem. Wien:MUW;2014.

12. Auer CM. Das Team rund um den Hausarzt - Konzept zur multiprofessionellen und interdisziplinären Primärversorgungin Österreich. Wien:BfG; 2014. 
13. Kringos DS, Boerma WG, Bourgueil Y, Cartier T, Hasvold T, Hutchinson A, et al. The European primary care monitor: structure, process and outcomeindicators. BMCFam Pract. 2010;11:81.https://doi.org/10.1186/1471-2296-11-81.

14. Starfield B, Starfield B. Primary care assessment tool: primary care assessment tool. 2017. http://www.jhsph.edu/ pcpc/pca_tools.html.Zugegriffen:4. Mai2017.

15. Pasarín MI, Berra S, González A, Segura A, Tebé C, GarcíaAltés A, et al. Evaluation of primary care: the "primary care assessment tools-facility version" for the Spanish health system. Gac Sanit. 2013;27(1):12-8. https://doi.org/ 10.1016/j.gaceta.2012.03.009.

16. De Rosis S, Seghieri C. Basic ICT adoption and use by general practitioners: an analysis of primary care systems in 31 European countries. BMC Med Inform Decis Mak. 2015;15:70.https://doi.org/10.1186/s12911-015-0185-z.
17. Rumball-Smith J, Wodchis WP, KonéA, KenealyT, BarnsleyJ, Ashton T. Under the same roof: co-location of practitioners within primary care is associated with specialized chronic care management. BMC Fam Pract. 2014;15:149. https:// doi.org/10.1186/1471-2296-15-149.

18. Samarasundera E, Walsh T, Cheng T, Koenig A, Jattansingh $\mathrm{K}$, Dawe A, et al. Methods and tools for geographical mapping and analysis in primary health care. Prim Health Care Res Dev. 2012;13(1):10-21. https://doi.org/10.1017/ S1463423611000417.

19. Hoffmann K, Wojczewski S, George A, Schäfer WL, Maier M. Stressed and overworked? A cross-sectional study of the working situation of urban and rural general practitioners in Austria in the framework of the QUALICOPC project. Croat Med J. 2015;56(4):366-74. https://doi.org/10.3325/ cmj.2015.56.366. 